\title{
Human time estimation: Procedural effects
}

\author{
MICHEL GUAY and ALAN W. SALMONI \\ Laurentian University, Sudbury, Ontario, Canada
}

\section{(Lawrence M. Ward, Sponsor)}

\begin{abstract}
The purpose of this experiment was to investigate the effects of various trial procedures on human time estimation. Four different procedures were used: (1) experimenter-paced intertrial interval/experimenter-paced retention interval, (2) self-paced intertrial interval/experimenterpaced retention interval, (3) experimenter-paced intertrial interval/self-paced retention interval, and (4) self-paced intertrial interval/self-paced retention interval. Auditory time lengths of 1, 4, and $8 \mathrm{sec}$ were estimated by the method of reproduction. Results showed that the two self-paced retention interval conditions produced a typical range effect in that the 1 -sec criterion duration was overestimated and the 4- and 8-sec durations were underestimated. In addition, the two homogeneous trial conditions (i.e., procedures 1 and 4 above) produced more accurate time estimation performance than did the two heterogeneous conditions. Variable error increased as the duration of the time to be estimated increased, and subjects were more variable under the two experimenter-paced retention interval conditions than under the two self-paced conditions. We concluded that the self-paced intertrial interval/self-paced retention interval condition seems to be the best procedure to maximize time-estimation performance.
\end{abstract}

Although past research in human time estimation was done by experimental psychologists, more recently this research has been carried out by researchers interested in human movement, because time estimation seems to have relevance to the coordination of movement. Obviously, the durations to be estimated are of major interest in this research, but there is also some evidence that different procedures may have different effects on time estimation (Buckolz \& Gervais, 1976; Buckolz \& Guay, 1975; Treisman, 1963). Thus it appeared to us that a systematic study of trial interval procedures was warranted, and the main purpose of the present research was to investigate the potential effects of experimenter-paced versus self-paced trial intervals.

A common finding in experiments using experimenterpaced trial intervals is that subjects' time estimates get longer over blocks of trials. This has been referred to as the lengthening effect. For example, Warm, Morris, and Kew (1963), who used the method of reproduction for auditory durations of $0.5,1,5$, and $10 \mathrm{sec}$, found a statistically reliable increase in magnitude of reproduction judgments when comparing the first trial with the last 10 trials. These authors interpreted the lengthening effect as an increase in boredom during the repetitive performance of a nonrewarding task. Treisman (1963), who also used experimenter-paced intertrial and retention intervals, found a similar lengthening effect over blocks of trials. He interpreted this effect as being due to changes in specific temporal arousal, which was the central construct in his model of the internal time-keeping mechanism. Thus, in the older literature, this effect was given theo-

Requests for reprints should be sent to M. Guay, School of Human Movement, Laurentian University, Sudbury, ON P3E 2C6, Canada. retical importance. More recently, however, several studies (e.g., Buckolz \& Gervais, 1976; Buckolz \& Guay, 1975) seemed to indicate that this lengthening effect might not occur when self-pacing of the trial intervals was employed. A self-paced intertrial interval/self-paced retention interval, whereby the subject controlled the onset of the criterion time duration to be estimated and the length of the retention interval before recall of the criterion, was employed in these studies.

One might question, therefore, the theoretical importance placed on this lengthening effect and also whether the self-pacing of the intertrial interval and/or the retention interval was responsible for the elimination of the effect. To enable us to answer these questions, two additional conditions were combined with those outlined above: self-paced intertrial interval/experimenter-paced retention interval and experimenter-paced intertrial inter$\mathrm{val} /$ self-paced retention interval. Consequently, the purpose of the present study was to determine the effects of the four different trial procedures on time estimation. This study seemed warranted because all of these trial procedures have not been systematically manipulated in one experiment to date. On the basis of the previous studies, a lengthening effect was expected in the experimenterpaced intertrial interval/experimenter-paced retention interval condition but not in the totally self-paced condition. The effects of the other two trial interval conditions could not be predicted, but we felt that the results of these two conditions would shed light on the interval responsible for the lengthening effect and would indicate of what theoretical importance this might be to time estimation. In addition, we expected the results of the present research to indicate which trial procedure to use to maximize timeestimation performance. 
To test the above ideas, we employed the method of reproduction, and the subjects were required to refrain from using any time-estimate-aiding strategies such as covert counting. The subject complied, as validated by extensive questioning of each subject after the testing was completed. Auditory durations of 1,4 , and $8 \mathrm{sec}$ were presented to and estimated by the subjects over blocks of trials. Taking past research (e.g., Guay, 1982) into consideration, we decided that the experimenter-paced intertrial interval and retention interval should be $10 \mathrm{sec}$, which represented an average of intervals used in past work.

\section{METHOD}

\section{Subjects}

The subjects for this research were 12 volunteer undergraduate students. Their average age was 21.9 years.

\section{Apparatus and Task}

The time estimation/reproduction task required that either the experimenter or the subject initiate the onset of the time duration (criterion) to be estimated. Following presentation of the criterion, either the experimenter or the subject initiated the attempted reproduction of the criterion duration.

The subject sat facing a table upon which two microswitches were placed $20 \mathrm{~cm}$ apart. Both switches were connected to a DEC PDP-11/03 computer. The left switch was used in the subject-paced conditions to allow the subject to control the length of the intertrial and retention intervals. The right microswitch was operated by the subject to estimate the criterion duration. As in the computer presentation of the criterion, a tone sounded when the subject activated the right microswitch and was turned off when the subject released the switch. The tones were presented to the subject through Sony DR-7 headphones, and all intervals that were not self-paced were controlled by the computer.

\section{Design}

As described above, there were four different trial interval conditions or procedures: (1) experimenter-paced intertrial interval/experimenterpaced retention interval, (2) self-paced intertrial interval/experimenterpaced retention interval, (3) experimenter-paced intertrial interval/selfpaced retention interval, and (4) self-paced intertrial interval/self-paced retention interval. In addition, subjects were required to estimate time durations of 1,4 , and $8 \mathrm{sec}$. Thirty-five trials were given under each trial interval condition, with the first five trials designated for practice. The above factors were combined to form a $4 \times 3 \times 6$ (conditions $\times$ durations $\times$ blocks) design with repeated measures on all factors.

\section{Procedure}

Subjects attended three separate sessions of 40 min each. The 12 treatment conditions were assigned to the subjects, with the order of occurrence randomized and counterbalanced across subjects. In any testing session, four trial interval conditions were employed, but the duration to be estimated was constant within a session.

The single trial procedure for the experimenter-paced/experimenterpaced condition was the following: a constant 10 -sec intertrial interval, presentation of the criterion duration, a constant 10 -sec retention interval, and recall of the criterion (this interval was initiated by the experimenter and terminated by the subject). The procedure for a single trial in the self-paced/self-paced condition was the following: a selfpaced intertrial interval, presentation of the criterion (the onset of which was initiated by the subject), a self-paced retention interval, and recall of the criterion duration (initiated and terminated by the subject). The other two trial interval conditions were a combination of these procedures. During the instruction phase, accuracy of performance was stressed and the subject was asked to demonstrate his/her understanding of the task. In addition, the subject was asked not to use a timeestimate-aiding strategy at any time and was questioned at the end of the treatment condition to ensure that these instructions had been car- ried out. (Indeed, no subjects reported the use of any time-estimate-aiding strategies.)

\section{Data Analysis}

The durations of the criterion estimates, self-paced intertrial intervals, and self-paced retention intervals were recorded by the computer. Constant error (accuracy) and variable error (consistency) were computed over five-trial blocks and were used in the $4 \times 3 \times 6$ analysis of variance with repeated measures on all factors.

\section{RESULTS}

\section{Constant Error}

The main effect of condition was significant $[F(3,33)$ $=13.94, p<.01]$. The condition $\times$ criterion duration interaction was also significant $[F(6,66)=9.32$, $p<.01]$. The results in Table 1 show that the two selfpaced retention interval conditions generally caused an underestimation of the 4- and 8-sec criterion durations, whereas the two experiment-paced conditions caused an overestimation of these durations. No such trend was present for the 1-sec duration condition. More specifically, a Duncan test $(p<.05)$ of the simple effects indicated that the self-paced/experimenter-paced procedure was significantly different from the other three procedures for the 4- and 8-sec durations. That is, for the 4- and 8-sec durations, subjects overestimated under the selfpaced/experimenter-paced procedure and underestimated under the other three procedures. In addition, the experimenter-paced/experimenter-paced condition was significantly different from the two self-paced retention interval conditions for the 8-sec duration. That is, for the 8 -sec duration, subjects overestimated under the selfpaced/experimenter-paced procedure and underestimated under the two self-paced retention interval conditions. Finally, the self-paced/self-paced condition was significantly different from the experimenter-paced/self-paced condition. That is, for the 8-sec duration, subjects underestimated to a lesser degree under the self-paced/self-paced procedure than the experimenter-paced/self-paced procedure. Along similar lines, the two homogeneous conditions (i.e., experimenter-paced/experimenter-paced and selfpaced/self-paced) led to more accurate time-estimation per-

Table 1

Mean Constant and Variable Error (in msec) for Durations and Conditions

\begin{tabular}{cccc}
\hline Duration & Procedure* & Constant Error & Variable Error \\
\hline $1 \mathrm{sec}$ & EP/EP & +65 & 110 \\
& SP/EP & -6 & 146 \\
& EP/SP & +150 & 110 \\
& SP/SP & +58 & 114 \\
$4 \mathrm{sec}$ & EP/EP & -28 & 436 \\
& SP/EP & +588 & 471 \\
& EP/SP & -314 & 326 \\
& SP/SP & -158 & 335 \\
$8 \mathrm{sec}$ & EP/EP & +256 & 858 \\
& SP/EP & +915 & 849 \\
& EP/SP & -894 & 704 \\
& SP/SP & -362 & 646 \\
\hline
\end{tabular}

*EP $=$ experimenter-paced. $\mathrm{SP}=$ self-paced. 
formance than did the two mixed conditions, except at the 1 -sec duration, where, as reported above, the four conditions did not result in significantly different estimates. No other main effects or interactions were significant.

\section{Variable Error}

The results for variable error were very similar to those reported above for constant error. As expected, subjects' estimates became more variable as the duration to be estimated increased, producing a significant duration main effect $[F(2,22)=58.85, p<.01]$. As can be seen in Table 1 , subjects' estimates were less variable in the two conditions with a self-paced retention interval than in the two experimenter-paced retention interval conditions, producing a significant trial procedures main effect $[F(3,33)=6.51, p<.01]$. Indeed, a post hoc analysis using the Duncan test $(p<.05)$ demonstrated that estimates were significantly more variable for the experimenter-paced/experimenter-paced and the selfpaced/experimenter-paced conditions than for the experimenter-paced/self-paced and self-paced/self-paced conditions. No other significant main effects or interactions were observed.

\section{Self-Paced Interval Times}

The mean for the self-paced intertrial interval under the self-paced/experimenter-paced condition was $2.3 \mathrm{sec}$, and the mean for the self-paced retention interval for the experimenter-paced/self-paced condition was $1.3 \mathrm{sec}$. Finally, the means for the self-paced intertrial interval and retention interval under the self-paced/self-paced condition were 1.7 and $1.1 \mathrm{sec}$, respectively. Obviously, the subjects were "comfortable" working at intervals much shorter than the 10-sec intervals used in the experimenterpaced conditions.

\section{DISCUSSION}

The primary purpose of this research was to determine whether different trial procedures had different effects on time-estimation ability. In terms of time-estimation accuracy (i.e., constant error), we found that estimations of time durations as short as $1 \mathrm{sec}$ were not affected by trial procedures; however, longer durations, such as 4- and 8-sec durations, were affected. Generally, for the 4- and 8-sec durations, a self-paced retention interval caused the subjects to underestimate the criterion durations, whereas the experimenter-paced retention intervals caused overestimation. More important, however, the homogeneous trial procedures (i.e., both the self-paced and experimenter-paced intertrial and retention intervals) afforded more accurate time estimations than did the mixed trial procedures. This effect is seen best by looking at the absolute magnitudes of the constant error scores for the 4- and 8-sec durations in Table 1. It may be that it is difficult for subjects to switch between selfpacing and experimenter pacing within a trial or that a consistent temporal rhythm for the different intervals is easier. For the present experiment, the self-paced/self-paced condition produced a 1-sec rhythm, the experimenter-paced/experimenter-paced condition produced a 10sec rhythm, and the mixed conditions produced 1-sec and 10-sec rhythms.

In terms of consistency of estimation over trials (i.e., variable error), the self-paced retention intervals afforded more consistent behavior than did the experimenter-paced retention intervals. This effect, as well as the fact that the self-paced retention intervals produced underestima- tions of the criterion and the experimenter-paced retention intervals caused overestimation, is possibly a memory phenomenon. Thus, the two conditions under a 10 -sec retention interval caused greater inconsistency and greater positive bias in the estimations of the criterion durations than the two conditions under a retention interval of approximately $1 \mathrm{sec}$. These trends are not out of line with retention interval effects in the motor short-term memory literature, which has shown that an increased retention interval often leads to a negative shift in constant error and an increase in variable error. In addition, as can be seen in Table 1, the two self-paced retention interval conditions produced a typical range effect in that the $1-\mathrm{sec}$ criterion duration was overestimated and the two longer criterion durations were underestimated. This range effect was not present for the two experimenter-paced retention interval conditions. Again, some type of retention interval "length effect" would seem to be the explanation.

A typical finding of earlier time-estimation studies (e.g., Treisman, 1963) was a lengthening effect, which was defined as an increase in the average of the estimated durations over blocks of trials. In the present research, however, neither the blocks main effect nor the block $\times$ procedure interaction was significant for constant error. Thus, there was no evidence for a lengthening of the estimated durations over trials, which is consistent with more recent studies in time estimation (e.g., Buckolz \& Gervais, 1976; Guay \& Wilberg, 1983). The present research seems to disprove the hypothesis put forward in these earlier studies that it was the self-pacing of the trial intervals that was responsible for eliminating the lengthening effect, since the lengthening effect was absent for both the self-paced and experimenter-paced conditions in the present study. The question remains as to why some research does and some does not find a lengthening effect.

One possible explanation has to do with the instructions given to the subjects. In the Treisman (1963) study, subjects were given the criterion duration at the start of each block of trials. This was not done in the present research. This suggests that the lengthening effect, when it occurs, may reflect response bias rather than that the lengthening effect is a property of a memorial process. Another possible reason for the absence of a lengthening effect is the number of trials. If the lengthening effect is due to boredom, as some researchers have proposed (e.g., Dewolfe \& Duncan, 1959; Kleiser, 1953), we might expect a greater lengthening effect with greater numbers of trials. Trials per se, however, does not seem to be the single cause, since subjects in the present study received 30 trials per criterion duration, whereas subjects in the Treisman (1963) and Warm et al. (1963) studies received 24 and 20 trials, respectively. (A lengthening effect was obtained in the latter two studies but not in the present research.) This in itself does not rule out boredom, since the experimental procedures could interact with the number of trials to produce a "boring" research setting for the subjects. Some support for this was provided by the introspective reports of the subjects obtained at the conclusion of the testing. Subjects generally reported being more motivated under the conditions requiring self-pacing than under the totally experimenter-paced condition. The present research procedures, which required subjects to understand and perform under four different conditions, may have been more challenging and therefore less boring. This was not the case in the Treisman (1963) and Warm et al. (1963) studies, in which only the experimenter-paced/experimenterpaced condition was employed. At this point, because of the inconsistent nature of the lengthening effect across studies, it would not seem to be of theoretical importance to the understanding of human time estimation.

In summary, it is difficult to determine from the present results which trial procedure maximizes time-estimation abilities; however, it is clear that a mixed format is not desirable, especially if accuracy of estimation is the primary goal. In terms of response accuracy, the two homogeneous trial procedures seem to be of equal efficacy. The procedure producing the most consistent performance will not be known until an experiment is run in which the experimenter-paced and self-paced retention intervals are of equal length. If it can be assumed that the subjects in the self-paced conditions responded at what they considered to be a comfortable pace, then relatively short trial intervals would seem to be optimal. Since there are obviously individual differences as to a "comfortable" pace, a totally self-paced procedure may be preferable. 


\section{REFERENCES}

Buckolz, E., \& GervaIs, F. (1976). Effects of verbal mediation with and without feedback on time estimation. Journal of Human Movement Studies, 2, 255-270.

Buckolz, E., \& GUAY, M. (1975). Effects of feedback and procedures on human time estimation. Journal of Human Movement Studies, 1 , 132-142.

Dewolfe, R. K. S., \& Duncan, C. P. (1959). Time estimation as a function of level of behavior of successive tasks. Journal of Experimental Psychology, 58, 153-158.

GUAY, M. (1982). Short-term retention of temporal auditory information. Perceptual \& Motor Skills, 55, 19-26.
GuAY, M., \& WiLberg, R. B. (1983). Short-term retention of temporal visual information. Perceptual \& Motor Skills, 56, 139-146.

KLEISER, J. R. (1953). The effect of habit formation, mental activity, and gross muscle movements on the reproduction of time. Dissertation Abstract, 13, 886.

Treisman, M. (1963). Temporal discriminations and the indifference interval: Implications for a model of the "internal clock." Psychological Monographs, 77(13, Whole No. 576).

Warm, J. S., MorrIs, J. R., \& KEw, J. K. (1963). Temporal judgement as a function of nosological classification and experimental method. Journal of Psychology, 55, 287-297.

(Manuscript received for publication June 12, 1987.) 